\title{
Asymptotic Behaviour of Eigenfunctions for Multiparticle Schrödinger Operators
}

\author{
J. M. Combes \\ Département de Mathématiques, Centre Universitaire de Toulon, La Garde, France \\ L. Thomas \\ Département de Physique Théorique, Université de Genève, Genève, Suisse
}

Received February 2; in revised form May 14, 1973

\begin{abstract}
O'Connor's approach to spatial exponential decay of eigenfunctions for multiparticle Schrödinger Hamiltonians is developed from the point of view of analytic perturbations with respect to transformation groups.

This framework allows an improvement of his results in some directions; in particular if interactions are dilation analytic, exponential fall-off is shown to hold for any bound-state wave-function corresponding to an eigenvalue distinct from thresholds; it is shown that the exponential decay rate depends on the distance from the bound-state energy to the nearest threshold. Applications include non existence of positive energy bound-states.
\end{abstract}

Exponential fall off for eigenstates of many-particle Schrödinger Hamiltonians has been investigated in detail in a recent work of O'Connor [1]. He assumes that particles interact through multiplicative two-body potentials in $R+L_{\varepsilon}^{\infty}$ (Rollnik class plus arbitrarily small $L^{\infty}$ tail); then if $E_{0}$ is the lowest threshold of the system, bound-state wavefunctions associated to an eigenvalue $E$ with $E<E_{0}$, are in the domain of $e^{\theta \sqrt{2 M\left(E_{0}-E\right)} R}, 0<\theta<1$; here $M$ is the total mass of the system and $R$ is the radius of gyration operator about the centre of mass of the system. Other results on the asymptotic behavior of eigenfunctions for Schrödinger Hamiltonians include [1] Bazley and Fox, for one electron molecular ions; Faris, single particle Hamiltonians with exponentially decaying potentials; Alrichs, multiparticle atomic systems with Coulomb interactions.

These results supplement the smothness properties for multiparticle eigenfunctions described by Kato [2] and have important theoretical implications for bound-state or scattering problems. However they pertain to eigenfunctions with isolated eigenvalues of Schrödinger Hamiltonians with local interactions. These two constraints are not satisfactory from a mathematical point of view and not physically justified (e.g. existence of permutation or rotation symetries can lead to boundstates in the continuum).

Our major aim is to show how one can avoid such restrictions and obtain a general treatment of exponential fall-off with the help of analytic 
perturbation techniques developed in [3]. First by defining explicitly the concept of analyticity with respect to the boost group one can allow non strictly local potentials and prove pointwise ex ponential fall-off for the one particle density probabilities associated to the $\mathrm{N}$-particle isolated boundstate wave functions. Second since dilation analyticity allows to treat on an equal footing isolated bound-states and those enbedded in the continuum, one can expect to prove also exponential fall off for these last by requiring analyticity with respect to the linear group (boosts + dilations). We will develop this concept in the first section of the paper. In the second one we will use the techniques of [4] to prove the basic results on exponential fall-off, namely:

1) Bound state wave-functions associated to a non threshold eigenvalue $E$ are in the domain of $e^{\theta \sqrt{M\left|E-E_{\alpha}\right|+\Gamma_{\alpha}} R}$ where $E_{\alpha}$ is the nearest threshold and $\Gamma_{\alpha}=\left|\operatorname{Im} E_{\alpha}\right|$.

2) One particle probability densities have pointwise exponential fall-off.

Section III discusses applications of these concepts and results, in particular the question of the existence of positive energy bound-states recently treated by Simon [4] and an aspect of sudden perturbation theory for multiparticle systems which is illuminated by the concept of boost analyticity.

\section{Transformation Groups and Related Analytic Families}

The linear group $L_{N}$ is the set $\left\{(\tau, z) ; \tau \in \mathbb{R}^{3(N-1)} ; z \in \mathbb{R}^{+}\right\}$with the group law:

$$
(\tau, z) \times\left(\tau^{\prime}, z^{\prime}\right)=\left(z^{\prime-1} \tau+\tau^{\prime}, z z^{\prime}\right) .
$$

The unitary representation of $L_{N}$ on $H_{N} \approx L^{2}\left(R^{3(N-1)}\right.$ used here is given by:

$$
(u(\tau, z) \phi)(X)=z^{\frac{3(N-1)}{2}} e^{-i\langle X, z \tau\rangle} \phi(z X), \phi \in \mathscr{H},
$$

where $\langle\ldots\rangle$ is the scalar product on the configuration space $\mathbb{R}^{3(N-1)}$ as defined in the Appendix.

The two subgroups corresponding respectively to $\tau=0$ or $z=1$ are the usual dilation or boost groups. For any cluster decomposition of the $N$-particle system $D=\left\{C_{1}, C_{2}, \ldots, C_{k}\right\}$, the Hilbert space $\mathscr{H}_{N}$ factorizes as a tensor product.

$$
\mathscr{H}_{N}=\mathscr{H}^{C_{1}} \otimes \mathscr{H}^{C_{2}} \otimes \cdots \otimes \mathscr{H}^{C_{k}} \otimes \mathscr{H}^{D}
$$

where $\mathscr{H}^{C}$ is the state space for particles belonging to cluster $C$ (center of mass or $C$ separated) and $\mathscr{H}^{D}$ is the state space for the center of mass of clusters in $D$. Then the representation $u(\tau, z)$ also factorizes as

$$
u(\tau, z)=u^{C_{1}}(\tau, z) \otimes u^{C_{2}}(\tau, z) \cdots \otimes u^{C_{k}}(\tau, z) \otimes u^{D}(\tau, z)
$$


where $u^{C}(\tau, z)$ and $u^{D}(\tau, z)$ are defined as in (1) with $\phi \in \mathscr{H}^{C}$ or $\mathscr{H}^{D}$ respectively and where the position vectors $X_{C}$ or $X_{D}$ for particles in cluster $C$ or centers of mass of clusters in $D$ are substituted for $X$; then only the orthogonal projection of $\tau$ (for the scalar product $\langle.,$.$\rangle ) in the$ corresponding subspaces of configuration space is involved. In fact the decomposition of $u(\tau, z)$ comes from the direct sum decomposition of configuration space (A.5) associated to the cluster decomposition $D$.

To study the action of $u(\tau, z)$ on the $N$-particle kinetic energy operator $H_{0}$ we notice that in momentum representation one has

$$
\widetilde{(u(\tau, z) \phi)}(P)=z^{-\frac{3(N-1)}{2}} \tilde{\phi}\left(z^{-1} P+\bar{M} \tau\right), \quad \phi \in \mathscr{H},
$$

where $P$ is the $3(N-1)$ dimensional momentum vector and $\bar{M}$ is the mass operator defined in the appendix. In this representation $H_{0}$ is multiplication by the quadratic form $K(P)$ (A.6) so that simple computation gives:

$$
u(\tau, z) H_{0} u^{-1}(\tau, z)=z^{-2} H_{0}(z \tau)
$$

where $H_{0}(z \tau)$ is the multiplication operator by $K(P+\bar{M} z \tau)$.

If we now take $(\tau, z) \in \mathbb{C}^{3(N-1)} \otimes(\mathbb{C}-\{0\})$, the right member of (25) defines an operator analytic function

$$
H_{0}(\tau, z)=z^{-2} H_{0}(z \tau) .
$$

Since the perturbation $K(P+\bar{M} z \tau)-K(P)$ is $H_{0}-\varepsilon$-bounded, elementary smallness arguments imply that $H_{0}(\tau, z)$ is closed on $\mathscr{D}\left(H_{0}\right)$; so $H_{0}(\tau, z)$ is an analytic family of type (A) [5]. The operator $H_{0}(z \tau)$ is unitarily equivalent to $H_{0}(i \operatorname{Im}(z \tau))$ through $u(\operatorname{Re}(z \tau), 1)$. So the spectrum of $H_{0}(\tau, z)$ is the image $P(\tau, z)$ under a dilation of scale $z^{-2}$ of the paraboloïd

$$
\left\{\zeta \in \mathbb{C} ; \operatorname{Re} \zeta \geqq-\frac{Q(\sigma)}{2} \text { and }|\operatorname{Im} \zeta|^{2} \leqq 2 Q(\sigma) \operatorname{Re}(\zeta)+Q^{2}(\sigma)\right\}
$$

where $\sigma=\operatorname{Im}(z \tau)$ and $Q(\sigma)$ is the quadratic form on configuration space defined in the appendix. To prove this statement we notice that

$$
K(P+i \bar{M} \sigma)=K(P)-Q(\sigma)+2 i \bar{M}^{-1 / 2} P \cdot \bar{M}^{1 / 2} \sigma .
$$

For fixed value of $\operatorname{Re} \zeta=K(P)-Q(\sigma)$. The maximum value of $\operatorname{Im} \zeta$ $=2 \bar{M}^{1 / 2} P \cdot \bar{M}^{1 / 2} \sigma$ when $P$ varies on the corresponding energy sphere is $K(P)^{1 / 2} \cdot Q(\sigma)^{1 / 2}$.

One can define in the same way analytic families $H_{0}^{C}(\tau, z)$ and $H_{0}^{D}(\tau, z)$ for any subsystem $C$ or cluster decomposition $D$, substituting in (4) and (5) $H_{0}^{C}$ and $H_{0}^{D}$ respectively for $H_{0}$, where $H_{0}^{C}$ and $H_{0}^{D}$ are respectively the internal kinetic energy operators for particles in cluster $C$ or centers of mass of clusters in $D$. 
Using the decomposition (3) one can see that their spectra are as above with $Q_{C}(\sigma)$ or $Q_{D}(\sigma)$ instead of $Q(\sigma)$.

The interacting system has an hamiltonian

$$
H=H_{0}+\sum_{i \neq j} V^{i j}
$$

We assume that the interactions $V^{i j}$ between particles $i$ and $j$ are symmetric $\Delta$ compact operators in $\mathscr{H}^{i j} \simeq L^{2}\left(\mathbb{R}^{3}\right)$, i.e. are compact operators from $\mathscr{D}(\Delta)$ to $L^{2}\left(\mathbb{R}^{3}\right)$. This assumption has fundamental consequences for the hamiltonian $H$, namely: $H$ is self-adjoint with domain $\mathscr{D}(H)=\mathscr{D}\left(H_{0}\right)$. The essential spectrum is the half-line $\left(E_{0}, \infty\right)(6)$ where $E_{0}$ is the lowest many-body threshold ie

$$
E_{0}=\inf _{D} \sum_{C \in D} \sigma_{d}^{C}
$$

The lower bound is taken over many cluster decompositions and $\sigma_{d}^{C}$ is the discrete spectrum for the hamiltonian $H^{C}$ describing the interacting subsystem $C$ :

$$
H^{C}=H_{0}^{C}+\sum_{\substack{i, j \in C \\ i \neq j}} V^{i j} .
$$

Under our assumptions $E_{0}$ is finite.

We now define a concept which will be basic to our investigations and will allow in particular to make precise statements about the spectral properties of $H$.

Definition. Let $G$ be a subgroup of the linear group. Denote by $\hat{G}$ the complexification of $G$. Let $u(\gamma), \gamma \in G$, be the representation of $G$ as defined above. A two-body interaction $V^{i j}$ is $G$-analytic in $\mathscr{V} \subset \hat{G}$ if the family

$$
V^{i j}(\gamma)=u(\gamma) V^{i j} u(\gamma)^{-1}, \quad \gamma \in G
$$

has an analytic extension from $\mathscr{V} \cap G$ to $\mathscr{V}$ as a $\Delta$-compact operator.

This assumption can in fact be weakened without altering results of this paper, provided quadratic forms are used in place of unbounded operators. Then "weak $G$-analyticity" defined as follows would be sufficient:

$V^{i j}(\gamma), \gamma \in \mathscr{V}$, is an analytic family of compact operators from the form domain for $\Delta$ to its dual space [for the topology induced by $L^{2}\left(\mathbb{R}^{3}\right)$ ].

Simon has shown [7] that $G$-analyticity implies weak $G$-analyticity. We refer to his paper for the extension of our methods to such perturbations.

Dilation analytic potentials are studied in detail in $[3,7,8]$. Homogeneous potentials $\mathrm{gr}^{-\beta}, 0<\beta<\frac{3}{2}$ (or 2 in the weak case), Yukawa potentials are dilation analytic. On the other hand all multiplicative relatively compact interactions obviously are boost-analytic since they 
are invariant under boost-transformations; a similar property holds e.g. for electromagnetic forces

$$
-\nabla^{2} \rightarrow-(\nabla-e A)^{2}
$$

or spin-orbit forces under suitable conditions ([9]); this comes from the very simple action of the boost group on momentum operators. So all multiplicative interactions and those depending linearly in the momentum which are dilation analytic also are analytic with respect to the linear group. Notice also that the class of multiplicative interactions weakly analytic with respect to the boost group contains those belonging to $R+L_{\varepsilon}^{\infty}$ (Rollnik class plus arbitrarily small $L^{\infty}$ tail) which is the class studied by O'Connor.

We can now define a family $H(\gamma), \gamma \in \mathscr{V}$, by:

$$
H(\gamma)=H_{0}(\gamma)+\sum_{i \neq j} V^{i j}(\gamma)
$$

The fundamental property is that if $\gamma \in G, \gamma_{0}$ and $\gamma_{0}+\gamma$ are in $\mathscr{V}$ then

$$
H\left(\gamma+\gamma_{0}\right)=u(\gamma) H\left(\gamma_{0}\right) u(\gamma)^{-1} \text {. }
$$

In fact our assumptions imply that $H(\gamma)$ is obtained from $H_{0}(\gamma)$ through the addition of an $H_{0}-\varepsilon$-bounded perturbation; so that for all $\gamma \in \mathscr{V}$ the operator $H(\gamma)$ is closed on $\mathscr{D}\left(H_{0}\right)$ and analytic on this domain [type (A)]; identity (7) simply expresses unicity of analytic continuation in $\mathscr{V}$. The investigation of the spectral properties of $H(\gamma)$ can then be made following exactly the same lines as in [3]. The basic facts are indicated below; we refer to $[3,7]$ for the detailed proofs.

Definitions.

$\sigma(\gamma)$ : spectrum of $H(\gamma)$,

$\sigma_{e}(\gamma)$ : essential spectrum of $H(\gamma)$,

$\sigma_{d}(\gamma)$ : discrete spectrum of $H(\gamma)$ (isolated eigenvalues with finite multiplicity).

For any subsystem $C$ we denote by $H^{C}(\gamma)$ the family defined as $H(\gamma)$ for the system $C$. For any cluster decomposition $D$ the operator

$$
H^{D}(\gamma)=H_{0}^{D}(\gamma)+\sum_{C \in D} H^{C}(\gamma)
$$

is closed on $\mathscr{D}\left(H_{0}\right)$. Let $\sigma^{C}(\gamma), \sigma^{D}(\gamma)$ denote the spectra of $H^{C}(\gamma), H^{D}(\gamma)$ respectively etc .... Then we have the following basic properties:

1) If $E \in \sigma_{d}\left(\gamma_{0}\right), \gamma_{0} \in \mathscr{V}$, then $E$ also is an eigenvalue of $H(\gamma)$ for any $\gamma$ belonging to the maximal open connected subset $\mathscr{D}\left(\gamma_{0}, E\right)$ containing $\gamma_{0}$ and contained in $\left\{\gamma \in \mathscr{V} ; E \notin \sigma_{e}(H(\gamma))\right\}$.

2) Let $P(E, \gamma)$ denote the finite dimensional projection operator for $H(\gamma)$ associated to the eigenvalue $E \in \sigma_{d}(\gamma)$. Then $P(E, \gamma)$ is analytic in $\mathscr{D}\left(\gamma_{0}, E\right)$.

3.a)

$$
\sigma_{e}(\gamma)=\bigcup_{D}\left(\sigma_{0}^{D}(\gamma)+\sum_{C \in D} \sigma_{d}^{C}(\gamma)\right)
$$


where the union is over all many-cluster decompositions and $\sigma_{0}^{D}$ is the spectrum of $H_{0}^{D}(\gamma)$.

3.b) $\sigma(\gamma)$ is contained in a sector with arbitrarily small opening angle and direction $\beta(\gamma)^{1}$.

\section{Sketch of the Proofs}

1) and 2) are consequences of unitary implementability through $u(\gamma)$ of the mapping $H\left(\gamma_{0}\right) \rightarrow H\left(\gamma+\gamma_{0}\right)$, if $\gamma$ is real, and analyticity of $H(\gamma)$. These two facts and basic results from analytic perturbation theory imply local invariance of discrete spectrum and from this our statements.

The proofs of 3.a) and 3.b) is inductive and uses the Weinberg equation (see e.g. $[3,10])$ :

$$
(H(\gamma)-z)^{-1}=D(\gamma, z)+I(\gamma, z)(H(\gamma)-z)^{-1} .
$$

For $N=2$ the statements are true since according to the relative compactness of the interaction, $\sigma_{e}(\gamma)$ coincides with the spectrum of $H_{0}(\gamma)$, i.e. $\mathscr{P}(\gamma)$ which satisfies the sectoriality assumption.

We assume now that they hold for any subsystem. Both $I(\gamma, z)$ and $D(\gamma, z)$ are finite sums of products like $\left(H^{D}(\gamma)-z\right)^{-1} V^{i j}(\gamma)$ where $D=\left\{C_{1}, C_{2}, \ldots, C_{k}\right\}, k \geqq 2$, is a cluster decomposition of the total system. Such factors are bounded and $z$-analytic in the complementary set of $\sigma^{D}(\gamma)$. So $I(\gamma, z)$ and $D(\gamma, z)$ are analytic in the complement of $\bigcup_{D} \sigma^{D}(\gamma)$ where the union is over all many-cluster decompositions. Now any $H^{D}(\gamma)$ has the following tensorial structure:

$$
\begin{aligned}
& H^{D}(\gamma)=H^{C_{1}}(\gamma) \otimes I_{\mathscr{H}} c_{2} \ldots \otimes I_{\mathscr{H} C_{k}} \otimes I_{\mathscr{H} D}+I_{\mathscr{H}} c_{1} \otimes H^{C_{2}}(\gamma) \otimes \\
& \cdots \otimes I_{\mathscr{H} D}+\cdots+I_{\mathscr{H}} c_{2} \otimes I_{\mathscr{H}} c_{2} \otimes \cdots \otimes H_{0}^{D}(\gamma) .
\end{aligned}
$$

All factors $H^{C}(\gamma)$ satisfy by assumption the sectoriality condition 3.b). The same property holds for $H_{0}^{D}(\gamma)$ whose spectrum is a paraboloïd. Then theorems on tensor products of closed operators (11) (12) imply

$$
\sigma^{D}(\gamma)=\sigma_{0}^{D}(\gamma)+\sum_{C \in D} \sigma^{C}(\gamma)
$$

From the induction assumption for $\sigma^{C}(\gamma)$ and sectoriality of $H_{0}^{D}(\gamma)$ for each cluster decomposition $D$, the obvious extension of (A.9) for $\gamma \neq 0$ gives finally:

$$
\sigma^{D}(\gamma)=\sum_{D^{\prime} \supset D}\left(\sigma_{0}^{D^{\prime}}(\gamma)+\sum_{C \in D^{\prime}} \sigma_{d}^{C^{\prime}}(\gamma)\right)
$$

1 A sector is a set $\left\{z \in C ; \mid \arg \left(e^{-i \beta\left(z-z_{0}\right)} \mid<\frac{\phi}{2}\right\} ; \phi\right.$ is the opening angle and $\beta$ the direction of the sector. The paraboloid $\mathscr{P}(\tau, z)$ is contained in sectors with arbitrarily small opening angles and direction $\beta(\tau, z)=e^{-2 i \arg z}$. 
It remains to use compactness of $I(\gamma, z)$; the relative compactness of all $V^{i j}(\gamma)$ implies compactness of $I(\gamma, z)$ far from $\bigcup_{D} \sigma^{D}(\gamma)$ and therefore by analytic continuation everywhere in the complement of this open connected set. Then analytic Fredholm theory allows to conclude that $(H(\gamma)-z)^{-1}$ is meromorphic according to (8) in the complement of $\bigcup_{D} \sigma^{D}(\gamma)$; the fact that this meromorphy domain is maximal comes from the cluster properties of the system and we refer to [4] for the proof of this fact.

Concerning the sectoriality of $H(\gamma)$ we notice that $\sigma_{d}(\gamma)$ consists of poles of $(I-I(\gamma, z))^{-1}$; since $\|I(\gamma, z)\|$ goes to zero as an inverse power of dist. $\left(z, \bigcup_{D} \sigma^{D}(\gamma)\right)$ it is sufficient to prove that $\sigma_{e}(\gamma)$ is contained in a sector with arbitrary small aperture and direction $\beta(\gamma)$. But this holds from the induction assumption for any $\sigma^{D}(\gamma)$ and then from simple geometrical arguments for the finite union of these sets.

Statements 1) and 2) can be extended under some circumstances to non-isolated eigenvalues. In particular if we choose for $G$ the dilation group one has ([3]):

Lemma 1. If two-body interactions are dilation analytic in a cone $C_{a}=\{z \in \mathbb{C},|\arg z|<a\}$, with $a<\frac{\pi}{2}$, and if $\psi$ is an eigenvector associated to a non-threshold eigenvalue of $H\left(\right.$ i.e. $\left.E \notin \bigcup_{D}\left(\sum_{C \in D} \sigma_{d}^{C}\right)\right)$ then $\psi(0, z)$ $=U(0, z) \psi, z \in \mathbb{R}^{+}$, has an analytic extension to $\mathbb{C}_{a}$ satisfying:

$$
H(0, z) \psi(0, z)=E \psi(0, z) .
$$

This lemma extends to the linear group as is shown later. We first apply above statements to the boost group and recover O'Connor's result.

Theorem 1. If in (6) two-body interactions are boost analytic and if $\psi \in \mathscr{H}_{N}$ satisfies $H \psi=E \psi, E<E_{0}=\inf \sigma_{e}(H)$, then $\psi \in \mathscr{D}\left(e^{\theta \sqrt{2 M\left(E_{0}-E\right)} R}\right)$ for any $\theta$ with $0 \leqq \theta<1$.

Proof. $E$ is an isolated eigenvalue with finite multiplicity of $H=H(0,1)$. According to 3.a) the essential spectrum of $H(\tau, 1)$ is the union of paraboloïds (directed along $\left.R^{+}\right) E_{\alpha}+\sigma_{0}{ }^{D}(\tau, 1)$ where $E_{\alpha} \in \sum_{C \in D} \sigma_{d}^{C}(\tau, 1)$. So $E$ remains in the complement of such a paraboloïd as long as

$$
E<E_{\alpha}-\frac{1}{2} Q_{D}(\operatorname{Im} \tau) .
$$

Since $E_{0}<E_{\alpha}$ and from (A.3) this holds a fortiori if

$$
E<E_{0}-\frac{1}{2} Q(\operatorname{Im} \tau) .
$$


By 1) and 2) $\psi(\tau, 1)=e^{i\langle x, \tau\rangle} \psi$ has an analytic extension to the open domain

$$
\left\{\tau ; Q(\operatorname{Im} \tau)<2\left(E_{0}-E\right)\right\} .
$$

This implies that $\psi \in \mathscr{D}\left(e^{-\langle x, \sigma\rangle}\right)$ for all $\sigma$ with $Q(\sigma)<2\left(E_{0}-E\right)$. From this the theorem follows (see [1] O'Connor's thesis p. 64).

Singularities of the potential $\sum_{i \neq j} V^{i^{i j}}$ prevent the boundstate wavefunction to have in general pointwise exponential decay in any direction of configuration space. However such a property holds for "one-particle like" density probabilities associated to bound-state wave-functions, i.e. those concentrated on a 3-dimensional subspace of $\mathbb{R}^{3(N-1)}$, reducing the mass operator to a constant $\mu$.

To each particle or to each pair of disjoint clusters there corresponds such a subspace, its vectors describing the position of this particle or the relative position of the centers of mass associated to clusters. The density probability on $F$ associated to a state $\psi$ is then given by

$$
\xi(x)=\int_{F^{\perp}}|\psi(x, y)|^{2} d y
$$

where $F^{\perp}$ is the orthogonal complement of $F$ (for the scalar product $\langle.,$.$\rangle ).$

Corollary 1. Let $\psi$ be as in Theorem 1. Then any density probability $\xi$ associated to $\psi$ on a one-particle subspace with mass $\mu$ satisfy estimates

$$
e^{2 \theta \sqrt{2 \mu\left(E_{0}-E\right)}|X|} \xi(X)<C(\theta), \quad 0 \leqq \theta<1 .
$$

Proof. If $Q(\sigma)<2\left(E_{0}-E\right)$ then $\psi(i \sigma, 1)$ is an eigenstate of $H(i \sigma, 1)$ with eigenvalue $E$. Since $\mathscr{D}(H(i \sigma, 1))=\mathscr{D}\left(H_{0}\right)$ it is known $([13])$ that one particle like density probabilities associated to $\psi(i \sigma, 1)$ are continuous and bounded, their bound depending only on $\left\|H_{0} \psi(i \sigma, 1)\right\|$. For $\sigma \in F$, the density probability on $F$ for $\psi(i \sigma, 1)$ simply is $e^{-2\langle x, \sigma\rangle} \xi(X)$. So for all $\sigma F$ with $Q(\sigma)<2\left(E_{0}-E\right)$ one has

$$
e^{-2\langle x, \sigma\rangle} \xi(X)<C(\sigma) .
$$

Let $0 \leqq \theta<\theta^{\prime}<1$. Then there exists a finite set of cones, $C\left(\theta^{\prime}, \sigma_{i}\right)$ $=\left\{X \in \mathbb{R}^{3} ;\left\langle X, \sigma_{i}\right\rangle \geqq \theta^{\prime} Q^{1 / 2}(X) Q^{1 / 2}\left(\sigma_{i}\right)\right\}$ whose union is $\mathbb{R}^{3}$. Choosing $Q\left(\sigma_{i}\right)$ sufficiently close to $E_{0}-E$ and using the identity $Q(X)=\mu|X|^{2}$, $X \in F$, the corollary follows.

If one has bound-states in the continuum the preceding treatment does not work. However results from [3] suggest that non-threshold boundstates share most of the properties of isolated bound-states. In fact we prove below, under an additional analyticity assumption, that exponential decay still holds in a similar form, namely:

Theorem 2. If in (6) two-body interactions are $L_{N}$-analytic in a domain $\mathbb{C}^{3(N-1)} X \mathbb{C}_{a}$ (where $C_{a}$ is the cone defined in Lemma 1) and if $\psi \in \mathscr{H}_{N}$ 
satisfies $H \psi=E \psi$ where $E$ is a non-threshold eigenvalue, then for any $\theta$ with $0 \leqq \theta<1, \psi \in \mathscr{D}\left(e^{\theta \sqrt{2 M(}\left(\left|E-E_{\alpha}\right|+\Gamma_{\alpha}\right) R}\right.$ where $E_{\alpha}$ is the closest boundstate or resonance threshold and $\Gamma_{\alpha}=\left|\operatorname{Im} E_{\alpha}\right|$.

The proof of Theorem 2 requires the following auxiliary lemma which states that the analytic extension $\psi(0, z)$ defined in Lemma 1 has exponential fall-off; in fact for $\arg (z) \neq 0$ the vector $\psi(0, z)$ is an eigenvector of $H(0, z)$ associated to the eigenvalue $E$, which by our assumptions is isolated and has finite multiplicity ([3]). So we can expect to carry over for $\psi(0, z)$ the results of Theorem 1 . For convenience (but this is not essential) we will restrict ourselves to

$$
z=\varrho e^{ \pm i \frac{\pi}{4}}, \quad \varrho \in \mathbb{R}^{+} .
$$

Lemma 2. Let $\psi$ be as in Theorem 1 and assume $\frac{\pi}{4}<a$. Then for $\varrho \in R^{+}$the vector valued function $\psi\left(\tau, \varrho e^{i \frac{\pi}{4}}\right)$ is defined and analytic in

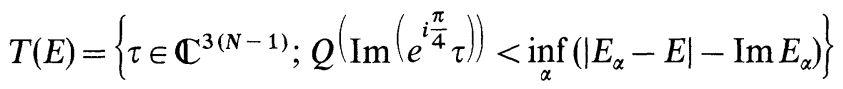

where the infimum is taken over all bound-state and resonance thresholds for $H\left(0, e^{i \frac{\pi}{4}}\right)$.

Proof. By Lemma $1, \psi\left(0, \varrho e^{i \frac{\pi}{4}}\right), \varrho \in \mathbb{R}^{+}$, is an eigenvector of $H\left(0, \varrho e^{i \frac{\pi}{4}}\right)$ associated to the finitely degenerate eigenvalue $E$. One has

$$
\psi\left(\tau, \varrho e^{i \frac{\pi}{4}}\right)=u(0, \varrho) \psi\left(\tau, e^{i \frac{\pi}{4}}\right)
$$

for any $\tau$ in the analyticity domain for $\psi\left(\tau, e^{i \frac{\pi}{4}}\right)$ which is given by

$$
\left\{\tau \in \mathbb{C}^{3(N-1)} ; \quad E \notin \bigcup_{D}\left(\sigma_{0}{ }^{D}\left(\tau e^{i \frac{\pi}{4}}\right)+\sum_{C \in D} \sigma_{d} C\left(\tau e^{\left.i \frac{\pi}{4}\right)}\right)\right\} .\right.
$$

Now $\sigma_{0}^{D}\left(\tau e^{i \frac{\pi}{4}}\right)$ is the paraboloind

$$
\left\{\zeta \in \mathbb{C} ; \operatorname{Im} \zeta \leqq \frac{Q_{D}(\sigma)}{2} \text { and }|\operatorname{Re} \zeta|^{2} \leqq-2 Q_{D}(\sigma) \cdot \operatorname{Im} \zeta+Q_{D}^{2}(\sigma)\right\}
$$

where $\sigma=\operatorname{Im}\left(\tau e^{i \frac{\pi}{4}}\right)$.

Let $E_{\alpha} \in \bigcup_{D}\left(\sum_{C \in D} \sigma_{d}^{C}\left(\tau e^{i \frac{\pi}{4}}\right)\right)$; denote by $-\Gamma_{\alpha}$ the imaginary part of $E_{\alpha}$. One has $\Gamma_{\alpha} \geqq 0([4,8])$. The condition $E \notin E_{\alpha}+\sigma_{0}(i \sigma)$ is equivalent to

$$
-2 Q_{D}(\sigma) \Gamma_{\alpha}+Q^{2}{ }_{D}(\sigma)-\operatorname{Re}\left(E-E_{\alpha}\right)^{2}<0
$$

and it is satisfied if

$$
Q_{D}(\sigma)<\left|E_{\alpha}-E\right|+\Gamma_{\alpha} .
$$


So $\psi\left(\tau, \mathrm{e}^{i \frac{\pi}{4}}\right)$ is analytic in

$$
\mathscr{D}(E)=\bigcap_{D}\left\{\tau \in \mathbb{R}^{3(N-1)} ; Q_{D}(\sigma)<\inf _{\alpha}\left(\left|E_{\alpha}-E\right|+\Gamma_{\alpha}\right\}\right.
$$

where $\sigma=\operatorname{Im}\left(e^{i \frac{\pi}{4}} \tau\right)$ and the infimum is taken over all $E_{\alpha}, \sum_{C \in D} \sigma_{d}{ }^{C}(\sigma)$.
$\mathscr{D}(E)$ is non empty since it contains $T(E)$.

A similar result holds of course for $\psi\left(\tau, e^{-i \frac{\pi}{4}}\right)$.

Proof of Theorem 2. Let $\mathscr{K}$ denote the dense subset of $\mathscr{H}$ consisting of states having compact support in configuration space. Let $\phi \in \mathscr{K}$ and define:

$$
F_{\phi}(\tau, z)=(\phi, \psi(\tau, z)), \quad(\tau, z) \in \mathbb{R}^{3(N-1)} X \mathbb{R} .
$$

One has

$$
F_{\phi}(\tau, z)=(u(-z \tau, 1) \phi, \psi(0, z))
$$

so that by Lemma 1 this function can be analytically continued in $\mathbb{C}^{3(N-1)} X \mathbb{C}_{a}$. Furthermore for fixed $\tau$ and $z \in \mathbb{C}_{e^{\frac{\pi}{4}}}$ one has an estimate

$$
\left|F_{\phi}(\tau, z)\right|<C(\tau) e^{b(\tau)|z|}
$$

where $C(\tau)$ and $b(\tau)$ are positive constants depending on $\phi$ (and in particular on its support in configuration representation in which $u(-z \tau, 1)$ acts as multiplication by $e^{i\langle x, z \tau\rangle}$ ). Then according to Phragmen-Lindelöf theorem,

$$
\left|F_{\phi}(\tau, z)\right| \leqq \sup _{|\arg (z)|<\frac{\pi}{4}}\left|F_{\phi}(\tau, z)\right| .
$$

Uniticity of analytic continuation and Lemma 2 imply

$$
F_{\phi}\left(\tau, \varrho e^{ \pm i \frac{\pi}{4}}\right)=\left(\phi, \psi\left(\tau, \varrho e^{ \pm i \frac{\pi}{4}}\right)\right)
$$

for $\tau \in T(E)$. According to (10) one has then

$$
\left|F_{\phi}\left(\tau, \varrho e^{ \pm i \frac{\pi}{4}}\right)\right| \leqq\|\phi\|\left\|\psi\left(\tau, e^{ \pm i \frac{\pi}{4}}\right)\right\| .
$$

From this, definition (12) of $F_{\phi}$ and density of $\mathscr{K}$ in $\mathscr{H}$, it follows that $\psi(\tau, z)$ has an analytic extension to $T(E) \times \mathbb{C}_{e^{i \frac{\pi}{4}}}$.

Taking $z=1$ we obtain that $\psi \in \mathscr{D}\left(e^{-i\langle x, \tau\rangle}\right)$ provided $Q(\operatorname{Re} \tau+\operatorname{Im} \tau)$ $<2 \inf _{\alpha}\left|E_{\alpha}-E\right|$ where the infimum is taken over all thresholds. We can now conclude as in Theorem 1.

Corollary 2. Let $\psi$ be as in Theorem 2. Then any density probability $\xi$ associated to $\psi$ on a one-particle subspace with mass $\mu$ satisfy estimates

$$
e^{2 \theta \sqrt{2 \mu \inf \left(\left|E_{\alpha}-E\right|+\Gamma_{\alpha}\right)}|X|} \xi(X)<C(\theta), \quad 0 \leqq \theta<1 .
$$


Proof. As in Corollary 1, since analytic continuation of the equation

$$
H(\tau, 1) \psi(\tau, 1)=E \psi(\tau, 1)
$$

to the domain $T(E)$ exists by Lemma 2 and implies that $\psi(i \sigma, 1)$ is an eigenstate of $H(i \sigma, 1)$ for $Q(\sigma)<2 \inf _{\alpha}\left(\left|E-E_{\alpha}\right|+\Gamma_{\alpha}\right)$.

\section{Concluding Remarks}

i) Although our main interest is in the qualitative aspect of the results obtained above, we want to make some critical remarks about the numerical factor $\sqrt{2 \mu \inf _{\alpha}\left(\left|E-E_{\alpha}\right|+\Gamma_{\alpha}\right)}$ involved in Corollary 2 .

The first remark concerns the fact that for the sake of generality we have not considered the maximal analyticity domain $\mathscr{D}(E)$ given by $(11)$ for our bound-states wave-functions but the domain $T(E)$ defined in Lemma 2.

Define for each cluster decomposition $D$ and a one particle subspace $F$ :

$$
n_{D F}=\sup _{\substack{\sigma \in F \\ Q(\sigma)=1}} Q_{D}(\sigma)
$$

Let $\psi$ be a bound-state wave-function with energy $E$; then repeating the proof of Theorem 2 we obtain the following domain of definition for $\psi(i \sigma, 1), \sigma \in F$ :

$$
\mathscr{D}_{F}(E)=\bigcap_{D}\left\{\sigma \in F ; Q(\sigma)<2 n_{D F}{ }^{-1} \inf _{\alpha}\left(\left|E_{\alpha}-E\right|+\Gamma_{\alpha}\right)\right\}
$$

where the infimum is taken over $E_{\alpha} \in \sum_{C \in D} \sigma_{d}^{c}\left(0, e^{i \frac{\pi}{4}}\right)$, so that in Corollary 2 the factor $\inf _{\alpha}\left(\left|E-E_{\alpha}\right|+\Gamma_{\alpha}\right)$ can be replaced by

$$
d_{F}(E)=\inf _{D}\left(n_{D F}^{-1} \inf _{\alpha}\left(\left|E_{\alpha}-E\right|+\Gamma_{\alpha}\right) .\right.
$$

This result is in general better as can be seen on the following examples:

a) $D=\left\{C_{1}, C_{2}\right\}$ and $P_{F}<P_{C_{1}}$ : then $n_{D F}=0$. This example is particuculary relevant in the two-cluster continuum limit for $\sigma_{e}(H)$; then the rate of exponential fall-off is strictly greater than $\left(\mu\left|E_{0}-E\right|\right)^{1 / 2}$ for a bound-state below the continuum.

b) Assume a three particle system, $D=\{12,3\}$ and $F$ is the subspace associated to the relative position of particles 2 and 3 . Then

$$
n_{D F}=1-\frac{m_{1} m_{3}}{\left(m_{1}+m_{2}\right)\left(m_{2}+m_{3}\right)}<1 .
$$


As an application assume a repulsive interaction between the identical particles 2 and 3 and only one bound-state for an attractive interaction between 1 and 2 (or 3 ) with energy $E_{0}$; then the rate of exponential decay for $\xi\left(X_{2}-X_{3}\right)$ associated to a three-particle bound state wave-function with energy $E<E_{0}$ is $\left(m_{2}\left(1-\frac{m_{1} m_{2}}{\left(m_{1}+m_{2}\right)} 2\right)^{-1}\left(E_{0}-E\right)\right)^{1 / 2}$

Another fact can still prevent these results from being optimal and is the possible existence of symmetries, either of the usual type (rotation, permutation, etc ...) or accidental like e.g. for the helium atom in the limit of zero interaction between electrons. We would expect that only some "compatible" thresholds contribute to the rate of fall-off for a bound-state having a given symmetry. However it is not always clear that the investigation developed in the paper can be carried out for the hamiltonian reduced on the subspace corresponding to this symmetry; in particular it is an open problem whether this reduced hamiltonian gives under the action of one of our groups an analytic family (see however [14] in the case of the dilation group). It is true in the case of the helium atom with no electronic repulsion and allows to recover the correct exponential fall-off for the known ground-state wave-functions, which is only very badly described by our general corollaries.

ii) It is interesting to compare the conclusion of the Theorem 2 with the Wigner-Von Neumann example [15] of a single particle Hamiltonian with spherically symmetric potential having a positive energy eigenvalue embedded in the essential spectrum. The eigenfunction corresponding to this eigenvalue vanishes like an inverse power of $r, r \rightarrow \infty$, and hence is surely not a boost analytic vector. But the potential is essentially of the form (trigonometric functions of $r$ ) $\times r^{-1}$ and is not dilatation analytic so Theorem 2 is not applicable.

In the appendix, we prove that for a single particle Hamiltonian with spherically symmetric potential $V(r)$ such that $V(r) \rightarrow 0 r \rightarrow \infty$, and $V(r)$ is continuous, $r>0$, boost analyticity of the eigenfunction $f_{E}$ implies the eigenvalue $E$ is $\leqq 0$. This result together with Theorem 2 provides a new proof of Simon's result [16] that single particle Hamiltonians with spherically symmetric dilatation analytic potential have no positive energy eigenvalues.

Recently Simon [4] has shown the non-existence of positive energy eigenvalues for $n$-particle Hamiltonians which have potentials dilatation analytic in the strip $\{\theta /|\operatorname{Im} \theta|<\pi / 2\}$ with continuous boundary values for $|\operatorname{Im} \theta|=\pi / 2$ by exploiting the boost analyticity of the eigenfunctions. (Such Hamiltonians include those with Yukawa and Coulomb interactions.) In outline, his argument runs as follows; by induction one supposes that $H$ has no strictly positive thresholds since for $n=2$ the only threshold is at zero. Assume $\psi \in L^{2}\left(\mathbb{R}^{3 n-3}\right)$ satisfies $H \psi=E \psi$, 
$E>0$; then $\psi$ satisfies the hypotheses of Theorem 2. Let $g \in C_{0}^{\infty}\left(\mathbb{R}^{3 n-3}\right)$ with supp $g \subset\{x /|x| \geqq R(g)>0\}$ and define $F(Z)=Z^{3 N} \int g(x) \psi(Z x) d^{3 N-3} x$, $Z \in(0, \infty)$. Then one easily shows that $F(Z)$ is regular in $D=\{Z|| \arg Z \mid$ $\leqq \pi / 2,|Z|>0\}$, is polynomially bounded in $D$, and (using the boost) falls off exponentially along the imaginary axes. Carlson's theorem then implies $F(Z)=0$. Since such $g$ 's are dense, $\psi=0$.

iii) In the appendix we discuss in detail a problem arising in sudden perturbation theory, where an $n$-particle system in an eigenstate $\psi_{0} e^{-i E t}, E<0$ is suddenly subjected to a homogeneous electric field (Stark effect) which is turned on in the time $0<t<T$. We show the existence of a solution $\psi_{T}(t), t>0, \psi_{T}(0)=\psi_{0}$, to the resulting Schrödinger equation with time dependent Hamiltonian which satisfies $\lim _{T \rightarrow 0} \psi_{T}(T)=\psi_{0}$. If $\psi_{0}$ is boost analytic then the transition amplitude $\left(\psi_{0}, \psi_{T}(t)\right)$ is differentiable in time $t$.

Acknowledgments. We are indebted to A. O'Connor, W. Faris, D. Pearson and B. Simon for helpful contributions to this work, and to Prof. Jauch for making our collaboration possible at the Section de Physique of Geneva University. One of us (J. M. C) also wants to acknowledge Prof. R. Phillips for his kind and helpful hospitality during summer 72 at the Math. department of Stanford University where part of this work has been prepared.

\section{Appendix}

\section{i) Some Facts about N-particle Kinematics}

Let $X=\left(x_{1}, x_{2}, \ldots, x_{n}\right)$ be the $N$-particle position vector in configuration space corresponding to particle $i$ having position $x_{i}$ in a given reference frame. We define a bilinear form on configuration space

$$
\left\langle X, X^{\prime}\right\rangle=\sum_{i=1}^{N} m_{i} x_{i} \cdot x_{i}^{\prime}
$$

where $m_{i}$ is the mass of particle $i$ and $x_{i} \cdot x_{i}^{\prime}$ is the usual scalar product of the three dimensional vectors $x_{i}$ and $x_{i}^{\prime}$. One can write

$$
\left\langle X, X^{\prime}\right\rangle=X \cdot \bar{M} X^{\prime}
$$

where the dot denotes the usual scalar product and the mass operator $\bar{M}$ is defined by this equality. The norm associated to the bilinear form is the classical moment of inertia of the system; according to Huygens formula the moment of inertia of a system is the sum of the moment of inertia of the center of mass and moment of inertia about the center of mass. Then one has:

$$
\langle X, X\rangle=\left(\sum_{i=1}^{N} m_{i} x_{i}\right)^{2}+Q(X)
$$


where $Q(X)$ is the moment of inertia about the $N$-particle center of mass and is related to the radius of gyration $R$ about this center of mass by

$$
Q(X)=M R^{2}
$$

where $M=\sum_{i=1}^{N} m_{i}, R^{2}=\sum_{i=1}^{N} m_{i}\left(x_{i}-x_{C M}\right)$ and $x_{C M}=\frac{1}{M}\left(\sum_{i=1}^{N} m_{i} x_{i}\right)$ is the center of mass position vector.

Since we consider a system without external forces we will always assume that positions of particles are given in the center of mass system; accordingly the configuration space is isomorphic to $\mathbb{R}^{3(N-1)}$ onto which $Q(X)$ defines a non degenerate quadratic form. This form can be diagonalized in many ways by choosing suitable sets of relative coordinates for the $N$-particles. In particular for any cluster decomposition.

$D=\left\{C_{1}, C_{2}, \ldots, C_{k}\right\}$ one has a splitting

$$
Q(X)=Q_{D}(X)+\sum_{C \in D} Q_{C}(X)
$$

where $Q_{C}(X)$ is the moment of inertia of particles in cluster $C$ about their center of mass and $Q_{D}(X)$ is the moment of inertia for centers of mass of clusters in $D$.

We can then define the projection operators $P_{C}$ and $P_{D}$ on the orthogonal complement (for the scalar product defined in A.1) of the nullspaces for $Q_{C}$ and $Q_{D}$. Then to (A.3) there corresponds a similar splitting of this scalar product:

$$
\left\langle X, X^{\prime}\right\rangle=\left\langle X, P_{D} X^{\prime}\right\rangle+\sum_{C \in D}\left\langle X, P_{C} X^{\prime}\right\rangle
$$

and each vector $X \in \mathbb{R}^{3(N-1)}$ splits into an orthogonal sum

with $X_{D}=P_{D}$ and $X_{C}=P_{C} X$.

$$
X=X_{D}+\sum_{C \in D} X_{C}
$$

Let $D^{\prime}$ be another cluster decomposition; we write $D \subset D^{\prime}$ if clusters in $D^{\prime}$ are obtained by partitioning clusters in $D$. One has then by (A.3) $Q_{D}(X)<Q_{D^{\prime}}(X)$ and accordingly $P_{D}<P_{D^{\prime}}$.

All these facts have similar counterparts in momentum space. Let $P=\left(p_{1}, p_{2}, \ldots, p_{N}\right), \sum_{i=1}^{N} p_{i}=0$ denote the momentum vector of the $\mathrm{N}$-particle system in the center of mass reference frame and define

$$
\begin{aligned}
K(P) & =\sum_{i=1}^{N} \frac{p_{i}{ }^{2}}{2 m_{i}} . \\
& =\frac{1}{2}\left(P \cdot \bar{M}^{-1} P\right)
\end{aligned}
$$


The quadratic form $K(P)$ is the classical kinetic energy in the center of mass system. For each cluster decomposition $D$ it can be diagonalized as

$$
K(P)=K_{D}(P)+\sum_{C \in D} K_{C}(P)
$$

where $K_{D}(P)$ is the kinetic energy for centers of mass of clusters in $D$ and $K_{C}(P)$ is the internal kinetic energy for particles in $C$.

Let $H_{0}$ be the quantum mechanical observable associated to $K(P)$; in the configuration representation, where the Hilbert space $\mathscr{H}_{N}$ for the $N$-particle quantum system in the center of mass reference frame is simply $L^{2}\left(\mathbb{R}^{3(N-1)}\right), H_{0}$ is obtained by substituting $-i \frac{\partial}{\partial x}$ for the coordinate $p$ of the momentum vector, $x$ and $p$ being canonically conjugate variables; (A.7) implies a decomposition

$$
H_{0}=H_{0}{ }^{D}+\sum_{C \in D} H_{0}^{C}
$$

Now let $D^{\prime}$ be another cluster decomposition which $D \subset D^{\prime}$. Then if $D=\left\{C_{1}, C_{2}, \ldots, C_{k}\right\}$ one has $D^{\prime}=\left\{D\left(C_{1}\right), D\left(C_{2}\right), \ldots, D\left(C_{k}\right)\right\}$ where $D(C)$ is a cluster decomposition of $C$ which gives

$$
H_{0}^{D^{\prime}}=H_{0}{ }^{D}+\sum_{C \in D} H_{0}{ }^{D(C)}
$$

as an immediate consequence of (A.7).

ii) For the single particle Hamiltonian with spherically symmetric potential $V(r)$ such that $V(r) \rightarrow 0$ as $r \rightarrow \infty$ and $V(r)$ is continuous for $r>0$, the $x \cdot a$, analyticity, $a$ arbitrary, of an eigenfunction $f_{E}$ implies that the eigenvalue $E$ is $\leqq 0$. To prove this remark we use the following lemma:

Lemma. Let $f(t)$ be a non-trivial real solution to the equation

$$
-\frac{d^{2}}{d t^{2}} f(t)=\omega^{2}(t), f(t) t_{0} \leqq t<\infty
$$

where $\omega$ is a positive real continuous function satisfying

$$
\lim _{t \rightarrow \infty} \omega(t)=\omega_{0}>0
$$

Then $f$ is not identically constant in any open interval. The graph of $f(t)$ consists of a succession of "oscillations".

Beyond some point $t_{1}>t_{0}$ where $f\left(t_{1}\right)=0, f^{\prime}\left(t_{1}\right)>0$, each oscillation $C_{m}, m=1,2, \ldots$ is composed of successively alternating bumps $\left\{t \in C_{m} \mid f(t) \geqq 0\right\},\left\{t \in C_{m} \mid f(t)<0\right\}$. Let $t_{m}^{i}, i=1,2$ denote the boundary 
points of the bumps, namely the first zero $\left(f^{\prime}>0\right)$ and second zero $\left(f^{\prime}<0\right)$ of $C_{m}$. We have the estimates in $C_{m}$ :

$$
\begin{aligned}
& f(t) \geqq f^{\prime}\left(t^{1}{ }_{m}\right) \frac{\sin \omega_{\max }^{m}\left(t-t^{1}{ }_{m}\right)}{\omega_{\max }^{m}}, \quad 0 \leqq\left(t-t^{1}{ }_{m}\right) \leqq \pi / \omega_{\max }^{m} ; \\
& f^{\prime}\left(t^{2}{ }_{m}\right) \leqq f^{\prime}\left(t^{1}{ }_{m}\right)\left(1-2\left(\omega_{\min }^{m} / \omega_{\max }^{m}\right)^{2}\right), \\
& f(t) \leqq f^{\prime}\left(t^{2}{ }_{m}\right) \frac{\sin \omega_{\max }^{m}\left(t-t^{2}{ }_{m}\right)}{\omega_{\max }^{m}}, \quad 0 \leqq\left(t-t^{2}{ }_{m}\right) \leqq \pi / \omega_{\max }^{m} ; \\
& f^{\prime}\left(t^{1}{ }_{m+1}\right) \geqq f^{\prime}\left(t^{2}{ }_{m}\right)\left(1-2\left(\omega_{\min }^{m} / \omega_{\max }^{m}\right)^{2}\right), \\
& \omega_{\max }^{m}=\sup _{t \in C_{m}} \omega(t), \omega_{\min }^{m}=\inf _{t \in C_{m}} \omega(t) .
\end{aligned}
$$

where

We also have the estimates $\frac{2 \pi}{\omega_{\max }^{m}} \leqq t^{1}{ }_{m+1}-t^{1}{ }_{m} \leqq \frac{2 \pi}{\omega_{\min }^{m}}$, and for any $\alpha>0, \int_{t_{0}}^{\infty} e^{a t} f^{2}(t) d t=\infty$.

Proof. The proof of this lemma is not difficult, although somewhat tedious; we derive only the estimates. First suppose that we have two such differential equations (A.10)

$$
-\frac{d^{2}}{d t_{1}^{2}} f_{1}=\omega_{1}^{2} f_{1}, \quad-\frac{d^{2}}{d t^{2}} f_{2}=\omega_{2}^{2} f_{2}
$$

with the boundary conditions $f_{1}\left(t_{1}\right)=f_{2}\left(t_{1}\right)=0, f^{\prime}{ }_{1}\left(t_{1}\right)=f^{\prime}{ }_{2}\left(t_{1}\right)>0$ and $\omega_{1} \geqq \omega_{2}$. By multiplying the first differential equation by $f_{2}$ and the second by $f_{1}$ and subtracting, we obtain the equality,

$$
-\frac{d}{d t}\left(f_{2} \frac{d}{d t} f_{1}-f_{1} \frac{d}{d t} f_{2}\right)=\left(\omega_{1}{ }^{2}-\omega_{2}{ }^{2}\right) f_{1} f_{2} .
$$

The integral of this equation from $t_{1}$ to $t>t_{1}$ in a region where both $f_{1}$ and $f_{2}$ are non-negative gives

$$
f_{2} \frac{d}{d t} f_{1}-f_{1} \frac{d}{d t} f_{2} \leqq 0,
$$

which can be further integrated to show $f_{2} \geqq f_{1}$. The choices $\omega_{1}=\omega_{\max }^{m}$, $\omega_{2}=\omega$ in $C_{m}$ show $f(t)$ majorizes the sine function in $\pi / \omega_{\max }^{m}$, and give the estimate $\left(t^{2}{ }_{m}-t^{1}{ }_{m}\right) \geqq \pi / \omega_{\max }^{m}$. The choices $\omega_{1}=\omega, \omega_{2}=\omega_{\min }^{m}$ lead to $\left(t^{2}{ }_{m}-t^{1}{ }_{m}\right) \leqq \pi / \omega_{\min }^{m}$. The estimate on successive derivatives across a positive bump is obtained from

$$
\begin{aligned}
-f^{\prime}\left(t^{2}{ }_{m}\right)+f^{\prime}\left(t^{1}{ }_{m}\right)= & \int_{t^{1}{ }_{m}}^{t^{2}} \omega^{2}(s) f d s \\
\geqq & \left(\omega_{\min }^{m}\right)^{2} \int \frac{\sin \omega_{\max }^{m}\left(s-t^{1}{ }_{m}\right)}{\omega_{\max }^{m}} d s \\
& t^{1}{ }_{m}<s<t^{1}{ }_{m}+\pi / \omega_{\max }^{m} \\
= & 2\left(\omega_{\min }^{m} / \omega_{\max }^{m}\right)^{2} .
\end{aligned}
$$


Estimates in the negative bump regions are obtained similarly. Finally, let $\alpha>0$ be given. There exist numbers $0<\tau_{\min } \leqq \tau_{\max }<\infty$ such that $\tau_{\text {min }} \leqq t^{1}{ }_{m+1}-t^{1}{ }_{m} \leqq \tau_{\max }$ for all $m$. Choose $t_{\alpha}$ so large that $1<e^{\alpha \tau_{\text {min }}}\left(1-2\left(\omega_{\text {inf }} / \omega_{\text {sup }}\right)^{2}\right)^{4}$ where $\omega_{\text {inf }}=\inf _{t \geqq t_{\alpha}} \omega(t)$, and $\omega_{\text {sup }}=\sup _{t \geqq t_{\alpha}} \omega(t)$.

The estimates for $f$ imply that

$$
\begin{aligned}
\int_{C_{m}} e^{\alpha t} f^{2}(t) d t & \geqq e^{a t^{1}{ }_{m}}\left(\frac{\pi}{2}\right)\left(\omega_{\max }^{m}\right)^{-2}\left(\left(f^{\prime}\left(t^{1}{ }_{m}\right)\right)^{2}+\left(f^{\prime}\left(t^{2}{ }_{m}\right)\right)^{2}\right) \\
& \geqq e^{\alpha t^{1}{ }_{m}}\left(\frac{\pi}{2}\right)\left(\omega_{\max }^{m}\right)^{-2}\left(f^{\prime}\left(t^{1}{ }_{m}\right)\right)^{2}\left(1+\left(1-2\left(\omega_{\min }^{m} / \omega_{\max }^{m}\right)^{2}\right)^{2}\right) \\
& \geqq e^{\alpha t^{1}{ }_{m}} C_{0}\left(f^{\prime}\left(t^{1}{ }_{m}\right)\right)^{2} \equiv S_{m} .
\end{aligned}
$$

Now $S_{m+1} \geqq S_{m}$ for $t^{1}{ }_{m} \geqq t_{\alpha}$ so that

$$
\int_{t_{0}}^{\infty} e^{\alpha t} f^{2}(t) d t \geqq \sum_{m} S_{m}=\infty
$$

Proposition. Let $f_{E}$ be a real function satisfying the Schrödinger equation $-\Delta f_{E}+V f_{E}=E f_{E}$ in $\mathbb{R}^{3}$, where $E>0$ and where $V(r)$ is spherically symmetric and continuous in $r$ for $r>0$, and $V(r) \rightarrow 0$ as $r \rightarrow \infty$. Assume further that for some $\alpha>0,\left\|e^{\alpha r} f_{E}\right\|_{L^{2}}<\infty$. Then $f_{E} \equiv 0$.

Proof. It is no restriction to assume $f_{E}$ is an eigenfunction of the spherical Laplacian with eigenvalue $l(l+1)$. If $f_{E}$ were not identically zero for $r$ sufficiently large, $F(r)=r \int P_{l} f_{E} d \Omega\left(P_{l}\right.$ is a Legendre Function; integration is over a sphere of radius $r$ ) would satisfy the hypotheses of the lemma $\left(\omega^{2}=E-V-\frac{1}{r^{2}} l(l+1)\right)$ and so $\int e^{\alpha r} F^{2}(r) d r=\infty$, contrary to the assumption of the proposition. The theory of ordinary differential equations implies $F(r)$ can be uniquely continued into $r=0$ and thus vanishes throughout $\mathbb{R}^{3}$. (Cf. [17], particulary lemma $C$ of appendix and $[18,19,20]$.)

iii) Finally, we discuss a particular problem in sudden perturbation theory and the sudden approximation (Stark effect) which uses the analytic techniques developed in the first of the paper. Let

$$
K_{T}(t)=\left\{\begin{array}{lc}
H & t<0 \\
H+\frac{t}{T} X=H+\frac{t}{T} \sum_{i=1}^{n} e_{i} a \cdot x_{i}, \quad 0 \leqq t \leqq T \\
H+X & t>T
\end{array}\right\}
$$

be the time dependent Hamiltonian for the $n$-particle system with interaction $X$ which is suddenly turned on in the time $0 \leqq t \leqq T$. $H$ is 
given by equation (6) with $V^{i j}$ real. $K_{T}(t)$ is essentially self-adjoint on $C_{0}^{\infty}$ for each $t$. Let $\psi_{T}(t)$ be a solution to

$$
i \frac{d}{d t} \psi_{T}(t)=K_{T}(t) \psi_{T}(t)
$$

which for $t \leqq 0$ is an eigenstate of $H$, i.e. $\psi_{T}(t)=\psi_{0} e^{-i E t}, t \leqq 0$. The sudden approximation consists in approximating $\psi_{T}(T)$ for $T$ small by $\psi_{0}$ (21). To justify this approximation, one must establish that (A.12) has a solution $\psi_{T}(t), t \geqq 0$ and

$$
s-\lim _{T \rightarrow 0} \psi_{T}(T)=\psi_{0} .
$$

Existence of a solution to (A.12) is not immediate, however, because the domain of $K_{T}(t)$ is time dependent $0 \leqq t \leqq T$ and the usual existence theorems are not applicable. To avoid this problem we first consider the equation

$$
i \frac{d}{d t} V_{T}(t) \psi_{0}=H_{T}(t) V_{T}(t) \psi_{0}, \quad 0 \leqq t \leqq T
$$

for $V_{T}(t) \psi_{0},\left(V_{T}(0) \psi_{0}=\psi_{0}\right)$ with

$$
H_{T}(t)=e^{i \frac{t^{2}}{2 T} X} H^{-i \frac{t^{2}}{2 T} X}, \quad 0 \leqq t \leqq T .
$$

We may apply the following theorem which is a special case of those proved in $[22-24]$.

Theorem. Let $A(t)$ be a family of self-adjoint operators in a Hilbert space depending on the parameter $t \in\left(0, T_{0}\right)$ with domain $\mathscr{D}$ independent of $t$. Let $B(t)=((1+i A(t))(1+i A(0)))^{-1}$ be strongly continuously differentiable. ( $B$ and $\dot{B}$ are bounded). Let $\left\|B^{-1}\right\|<M$ and set $\int_{0}^{T_{0}}\|\dot{B}(t)\| d t=N$. Then there exists a unique bounded operator $V(t)$ strongly continuous in $t$, $0 \leqq t \leqq T_{0}$ with $V(0)=1$ such that

(i) Vleaves $\mathscr{D}$ invariant and satisfies the estimate

$$
\left\|(1+i A(t)) V(t)(1+i A(0))^{-1}\right\|<e^{M N} ;
$$

(ii) for $\phi_{0} \in \mathscr{D}, \phi(t)=V(t) \phi_{0}$ is strongly differentiable and satisfies $i \frac{d}{d t} \phi(t)=A(t) \phi(t) ; A(t) \phi(t)$ is strongly continuous in $t$.

The estimate can be found in [22].) Since $H_{T}(t)$ is type $A$ holomorphic, it satisfies the conditions of this theorem. In particular, $H_{T}(t)$ has time independent domain. Thus (A.14) has a unique solution.

By the definition of $H_{T}(t)$ and conclusion (i) of the theorem we have $V_{T}(t)$ satisfies a uniform estimate in $t$ and $T$,

$$
\left\|\left(1+i H_{T}(t)\right) V_{T}(t)(1+i H(0))^{-1}\right\|<C, \quad 0 \leqq t \leqq T<T_{0} .
$$


On integrating (A.14), we obtain

$$
\left\|V_{T}(T) \psi_{0}-\psi_{0}\right\|=\left\|\int_{0}^{T} d S H_{T}(S) V_{T}(S) \psi_{0}\right\|<C T\left\|(1+i H(0)) \psi_{0}\right\|,
$$

proving that

$$
s-\lim _{T \rightarrow 0} V_{T}(T) \psi_{0}=\psi_{0}
$$

Finally, let

$$
\psi_{T}(t)=e^{-i \frac{t^{2}}{2 T} X} V_{T}(t) \psi_{0}, \quad 0 \leqq t \leqq T .
$$

Then $\psi_{T}(t)$ clearly satisfies (A.13). $\psi_{T}(t)$ satisfies (A.12) in the weakened sense that if $\phi$ is a fixed vector in $\mathscr{D}(X) \cap \mathscr{D}(H)$, then $\left(\phi, \psi_{T}(t)\right)$ satisfies $i \frac{d}{d t}\left(\phi, \psi_{T}(t)\right)=\left(K_{T}(t) \phi, \psi_{T}(t)\right)$. In particular, if $\phi=\psi_{0}$ has isolated eigenvalue $t<0$, or eigenvalue isolated from threshold with $H$ dilatation analytic $t<0$, then $\psi_{0}$ will be in $\mathscr{D}(X) \cap \mathscr{D}(H)$ and thus the transition amplitude $\left(\psi_{0}, \psi_{T}(t)\right)$ will be differentiable in $t$.

\section{References}

1. O'Connor, A.: Exponential decay for the bound-state wave-functions of several particle systems. Princeton thesis, May 72 and Dublin advanced study institute Preprint 72

Bazley, N. W., Fox, D. W.: Intern. J. Quantum Chem. 3, 581 (1969)

Faris, W.G.: Quadratic forms and essential self adjointness, to appear in Helv. Phys. Acta Ahlrichs, R. : Asymptotic behavior of atomic bound state wave functions (Preprint 2, Theoretical Chemistry Group, University of Karlsruhe)

2. Kato, T.: Commun. Pure Appl. Math. 10, 151 (1957)

3. Aguilar, J., Combes, J.M.: Commun. math. Phys. 22, 269 (1971) Balslev, E., Combes, J. M.: Commun. math. Phys. 22, 280 (1971)

4. Simon, B.: Absence of positive eigenvalues in a class of multiparticle quantum systems. Marseille Preprint (73)

5. Kato, T.: Perturbation theory for linear operators. Berlin-Heidelberg-New York: Springer 1966

6. Combes, J. M.: Commun. math. Phys. 12, 283 (1969)

7. Simon, B.: Commun. math. Phys. 27, 1 (1972)

8. Simon, B.: Phys. Letters A-36, 23 (1971) and to appear in Annals of Mathematics

9. Balslev, E.: Math. Scand. 19, 193 (1966)

10. Hunziker, W.: H.P.A. 39, 451 (1966)

11. Ichinose, T.: On operators on tensor products of Banach spaces Nagoya University Preprint

12. Reed, M., Simon, B.: Tensor products of closed operators on Banach spaces. To appear in Journal of Functional Analysis

13. Kato, T.: T.A.M.S. 70, 195 (1951)

14. Balslev, E.: Schrödinger operators with symmetries. CPT, CNRS. Marseille Preprint (1972)

15. von Neumann, J., Wigner, E.: Z. Phys. 30, 465 (1929) 
16. Simon, B.: Resonances in $n$-body quantum systems with dilatation analytic potentials and the foundations of time-dependent perturbation theory. Ann. Math. to appear

17. Kato, T.: Commun. Pure Appl. Math. 12, 403 (1959)

18. Agmon, S.: Lower bounds for Schrödinger type equations. Proceeding of Tokyo International Conference on Functional Analysis and Related Topics, 1969

19. Agmon, S.: J. Analyse Math. 23, 1 (1970)

20. Simon, B.: Comm. Pure Appl. Math. 21, 531 (1968)

21. Messiah, A.: Quantum mechanics, Vol. II, 740. Amsterdam: North Holland Publishing Co. 1969

22. Kato, T.: J. Math. Soc. Japan 5, 208 (1953)

23. Kato, T.: Commun. Pure Appl. Math. 9, 479 (1956)

24. Yosida, K.: Functional analysis 424. Berlin-Heidelberg-New York: Springer 1968

L. E. Thomas

Département de Physique

Théorique

Université de Genève

$\mathrm{CH}-1211$ Genève 4, Switzerland

J. M. Combes

Centre Universitaire de Toulon

Château St. Michel

F-83130 La Garde, France 